\title{
Advances in research on stress and behavior: special issue, 2020
}

\author{
Julio Licinio ${ }^{1}$ \\ C Springer Nature Limited 2020
}

Given the wealth of outstanding papers we have recently received on stress and behavior, we have created our second special issue of 2020, focused on this fundamental interdisciplinary topic. This stress and behavior special issue brings together work by some of the most important groups in this field worldwide.

We start with a paper from the Niculescu's group on precision medicine for stress-related disorders [1]. We then look at the role of different endocrine axes in stress susceptibility, specifically the role of thyroid hormones [2], proopiomelanocortin (POMC) [3], and the hypothalamic-pituitary-adrenal (HPA) axis and its interactions with immunity [4]. The role of immune mediators in the stress response is further illustrated by the paper by Ben-Yehuda et al., showing that maternal Type-I interferon signaling adversely affects the microglia and the behavior of the offspring, accompanied by increased sensitivity to stress [5]. The emerging interface of the gut microbiome, depressive-like behaviors, and inflammatory processes is explored by Pearson-Leary et al. [6].

As expected, circuits involved in substance abuse are relevant to the stress response. This is highlighted in this issue by papers showing that hippocampal $\mu$-opioid receptors mediate stress-induced impairment of memory retrieval [7] and that the endogenous ligand of cannabinoid receptors, anandamide, has protective effects on stress and fear-related behaviors in humans and mice [8].

The key roles of interesting other stress-related pathways are demonstrated by several articles. The contribution of microRNAs in the response to stress and stressrelated memories is addressed by Torres-Berrio et al. [9] and Sillivan et al. [10]. Fox et al. provide evidence that dendritic remodeling of D1 neurons by RhoA/Rho-kinase

Julio Licinio

juliolicinio@gmail.com

1 State University of New York, Upstate Medical University, Syracuse, NY 13210, USA mediates depression-like behavior [11]. Jin et al. show that Ahnak scaffolds p11/Anxa2 complex and L-type voltage-gated calcium channel and thereby modulates depressive behavior [12]. Lei et al. provide new data that SIRT1 in forebrain excitatory neurons produces sexually dimorphic effects on depression-related behaviors and modulates neuronal excitability and synaptic transmission in the medial prefrontal cortex [13]. Of particular relevance to our hyperconnected world, in which light emitting devices are prevalent at sleep time, Walker et al. document here that acute exposure to low-level light at night is sufficient to induce neurological changes and depressive-like behavior [14]. Data from the Coronary Artery Risk Development in Young Adults Study presented by Cabeza de Baca et al. show that chronic psychosocial and financial burden accelerates 5-year telomere shortening [15].

The therapeutic outcomes of stress and selective serotonin reuptake inhibitor (SSRI) antidepressant treatment on corticostriatal neurons is explored by Sargin et al. [16]. They demonstrate that S100a10 corticostriatal neurons exhibit distinct serotonin responses and have increased excitability, reveal that prolonged social isolation disrupts the specific serotonin response which gets restored by chronic antidepressant treatment, and identify cell-typespecific transcriptional signatures in S100a10 neurons that contribute to serotonin responses.

Taken together these remarkable articles increase the breadth of our knowledge on the critically important interface of stress and behavior, while simultaneously substantially enhancing our understanding of new mechanisms and pathways by which stress modulates behavior. It is highly probable that some of these pathways are either redundant or related to one another, while others may be independent. Future work should examine the inter-relationships across multiple stress-related pathways that ultimately impact on behavior. 


\section{References}

1. Le-Niculescu H, et al. Towards precision medicine for stress disorders: diagnostic biomarkers and targeted drugs. Mol Psychiatry. 2019. https://doi.org/10.1038/s41380-019-0370-z

2. Martinez ME, et al. Thyroid hormone influences brain gene expression programs and behaviors in later generations by altering germ line epigenetic information. Mol Psychiatry. 2018. https:// doi.org/10.1038/s41380-018-0281-4

3. Qu N, et al. A POMC-originated circuit regulates stress-induced hypophagia, depression, and anhedonia. Mol Psychiatry. 2019. https://doi.org/10.1038/s41380-019-0506-1

4. Iob E, Kirschbaum C, Steptoe A. Persistent depressive symptoms, HPA-axis hyperactivity, and inflammation: the role of cognitiveaffective and somatic symptoms. Mol Psychiatry. 2019. https:// doi.org/10.1038/s41380-019-0501-6

5. Ben-Yehuda $\mathrm{H}$, et al. Maternal Type-I interferon signaling adversely affects the microglia and the behavior of the offspring accompanied by increased sensitivity to stress. Mol Psychiatry. 2019. https://doi.org/10.1038/s41380-019-0604-0

6. Pearson-Leary $\mathrm{J}$, et al. The gut microbiome regulates the increases in depressive-type behaviors and in inflammatory processes in the ventral hippocampus of stress vulnerable rats. Mol Psychiatry. 2019. https://doi.org/10.1038/s41380-019-0380-x

7. Shi MM, et al. Hippocampal micro-opioid receptors on GABAergic neurons mediate stress-induced impairment of memory retrieval. Mol Psychiatry. 2019. https://doi.org/10.1038/s41380-019-0435-Z

8. Mayo LM, et al. Protective effects of elevated anandamide on stress and fear-related behaviors: translational evidence from humans and mice. Mol Psychiatry. 2018. https://doi.org/10.1038/ s41380-018-0215-1
9. Torres-Berrio A, et al. MiR-218: a molecular switch and potential biomarker of susceptibility to stress. Mol Psychiatry. 2019. https:// doi.org/10.1038/s41380-019-0421-5

10. Sillivan SE, et al. MicroRNA regulation of persistent stressenhanced memory. Mol Psychiatry. 2019. https://doi.org/10.1038/ s41380-019-0432-2

11. Fox ME, et al. Dendritic remodeling of D1 neurons by RhoA/ Rho-kinase mediates depression-like behavior. Mol Psychiatry. 2018. https://doi.org/10.1038/s41380-018-0211-5

12. Jin J, et al. Ahnak scaffolds p11/Anxa2 complex and L-type voltage-gated calcium channel and modulates depressive behavior. Mol Psychiatry. 2019. https://doi.org/10.1038/s41380-0190371-y

13. Lei Y, et al. SIRT1 in forebrain excitatory neurons produces sexually dimorphic effects on depression-related behaviors and modulates neuronal excitability and synaptic transmission in the medial prefrontal cortex. Mol Psychiatry. 2019. https://doi.org/10. 1038/s41380-019-0352-1

14. Walker WH 2nd, et al. Acute exposure to low-level light at night is sufficient to induce neurological changes and depressive-like behavior. Mol Psychiatry. 2019. https://doi.org/10.1038/s41380019-0430-4

15. Cabeza de Baca $T$, et al. Chronic psychosocial and financial burden accelerates 5-year telomere shortening: findings from the Coronary Artery Risk Development in Young Adults Study. Mol Psychiatry. 2019. https://doi.org/10.1038/s41380-019$0482-5$

16. Sargin D, et al. Mapping the physiological and molecular markers of stress and SSRI antidepressant treatment in S100a10 corticostriatal neurons. Mol Psychiatry. 2019. https://doi.org/10.1038/ s41380-019-0473-6 